\title{
Dome C ultracarbonaceous Antarctic micrometeorites
}

\section{Infrared and Raman fingerprints ${ }^{\star}$}

\author{
E. Dartois ${ }^{1}$, C. Engrand ${ }^{2}$, J. Duprat ${ }^{2}$, M. Godard ${ }^{2}$, E. Charon ${ }^{2}$, L. Delauche ${ }^{2}$, C. Sandt ${ }^{3}$, and F. Borondics ${ }^{3}$ \\ ${ }^{1}$ Institut d'Astrophysique Spatiale (IAS), CNRS, Univ. Paris Sud, Université Paris-Saclay, 91405 Orsay, France \\ e-mail: emmanuel.dartois@ias.u-psud.fr \\ 2 Centre de Sciences Nucléaires et de Sciences de la Matière (CSNSM), CNRS/IN2P3, Univ. Paris Sud, Université Paris-Saclay, \\ 91405 Orsay, France \\ 3 Synchrotron SOLEIL, L'Orme des Merisiers, BP48 Saint Aubin, 91192 Gif-sur-Yvette Cedex, France
}

Received 6 June 2017 / Accepted 4 October 2017

\begin{abstract}
Context. UltraCarbonaceous Antarctic MicroMeteorites (UCAMMs) represent a small fraction of interplanetary dust particles reaching the Earth's surface and contain large amounts of an organic component not found elsewhere. They are most probably sampling a contribution from the outer regions of the solar system to the local interplanetary dust particle (IDP) flux.

Aims. We characterize UCAMMs composition focusing on the organic matter, and compare the results to the insoluble organic matter (IOM) from primitive meteorites, IDPs, and the Earth.

Methods. We acquired synchrotron infrared microspectroscopy ( $\mu$ FTIR) and $\mu$ Raman spectra of eight UCAMMs from the Concordia/CSNSM collection, as well as N/C atomic ratios determined with an electron microprobe.

Results. The spectra are dominated by an organic component with a low aliphatic $\mathrm{CH}$ versus aromatic $\mathrm{C}=\mathrm{C}$ ratio, and a higher nitrogen fraction and lower oxygen fraction compared to carbonaceous chondrites and IDPs. The UCAMMs carbonyl absorption band is in agreement with a ketone or aldehyde functional group. Some of the IR and Raman spectra show a $\mathrm{C} \equiv \mathrm{N}$ band corresponding to a nitrile. The absorption band profile from 1400 to $1100 \mathrm{~cm}^{-1}$ is compatible with the presence of $\mathrm{C}-\mathrm{N}$ bondings in the carbonaceous network, and is spectrally different from that reported in meteorite IOM. We confirm that the silicate-to-carbon content in UCAMMs is well below that reported in IDPs and meteorites. Together with the high nitrogen abundance relative to carbon building the organic matter matrix, the most likely scenario for the formation of UCAMMs occurs via physicochemical mechanisms taking place in a cold nitrogen rich environment, like the surface of icy parent bodies in the outer solar system. The composition of UCAMMs provides an additional hint of the presence of a heliocentric positive gradient in the $\mathrm{C} / \mathrm{Si}$ and $\mathrm{N} / \mathrm{C}$ abundance ratios in the solar system protoplanetary disc evolution.
\end{abstract}

Key words. comets: general - meteorites, meteors, meteoroids - interplanetary medium - protoplanetary disks - astrobiology methods: laboratory: solid state

\section{Introduction}

Micrometeorites represent the largest mass flux of extraterrestrial material falling on Earth (e.g. Love \& Brownlee 1993; Taylor et al. 1996; Duprat et al. 2006; Zolensky et al. 2006; Prasad et al. 2013; Engrand et al. 2017). They sample the interplanetary dust present in the inner solar system, a large fraction coming from Jupiter-family comets together with a contribution attributed to asteroids, and Kuiper Belt and Oort Cloud comets (e.g. Janches et al. 2006; Nesvorný et al. 2010, 2011; Poppe et al. 2011; Poppe 2016). The search for and collection of micrometeorites performed on the Antarctic continent has allowed the recovery of particles preserved from significant atmospheric entry heating and which still hold information from interplanetary dust. This information otherwise could only be obtained via sampling by space probes. Several countries are involved in these collecting expeditions (e.g. Nakamura et al. 2005; Duprat et al. 2007; Taylor et al. 2008; van Ginneken et al. 2012; Yabuta et al. 2012, 2017). The

\footnotetext{
* Part of the equipment used in this work has been financed by the French INSU-CNRS program "Physique et Chimie du Milieu Interstellaire" (PCMI).
}

Concordia/CSNSM collection contains thousands of Antarctic micrometeorites collected near the French-Italian Concordia station on Dome C during several campaigns performed since 1999 with the help and support of the French Polar Institute PaulÉmile Victor (IPEV). Details of the collecting methods can be found in Duprat et al. (2007). A particularly interesting subset in the collections is the fraction of micrometeorites arising from the outer parts of the solar system, known as Kuiper Belt and/or Oort Cloud comet contributions, which are largely undersampled by space missions. These dust particles contain the record of past formation and evolution in the outer solar system. UltraCarbonaceous Antarctic MicroMeteorites (UCAMMs, Duprat et al. 2010) and a subset of chondritic porous interplanetary dust particles (IDPs) are most probably of this class (e.g. Dobrica et al. 2010).

The UCAMMs are exceptionally organic-rich, in particular containing organic matter that is significantly larger than a micron in size (Dartois et al. 2013; Bardin et al. 2014; Engrand et al. 2015; Charon et al. 2017) that is not found in other types of extraterrestrial matter. The organic content of UCAMMs is high enough to avoid the requirement of prior chemical treatments to be studied in the infrared, and can be 


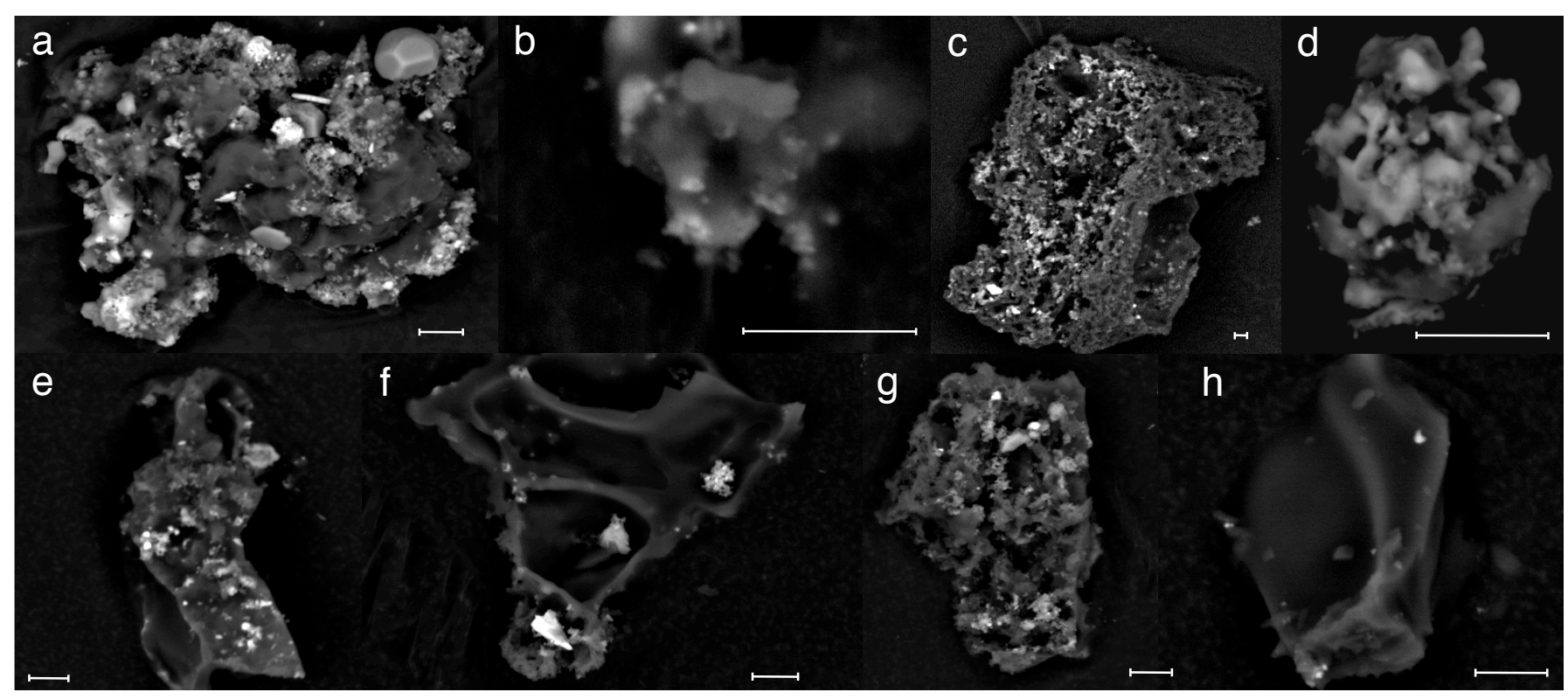

Fig. 1. Backscattered electron images recorded at $15 \mathrm{kV}$ of fragments of the UCAMMs samples analysed in this study: $a$ ) DC060919, $b$ ) DC060594, c) DC021119, d) DC060443, e) DC060718, f) DC060741, g) DC0609119, h) DC060565. Scale bars correspond to $5 \mu \mathrm{m}$ for each image. The unfragmented UCAMMS initial sizes are respectively: a) $79 \times 50 \mu \mathrm{m}, b) 66 \times 53 \mu \mathrm{m}$, c) $110 \times 87 \mu \mathrm{m}, d) 28 \times 24 \mu \mathrm{m}, e) 87 \times 53 \mu \mathrm{m}$, f) $200 \times 80 \mu \mathrm{m}, g) 275 \times 108 \mu \mathrm{m}, h) 44 \times 33 \mu \mathrm{m}$.

compared with the insoluble organic matter (IOM) extracted from meteorites for an insight into their similarities and differences. In this work, we report a $\mu$ FTIR and $\mu$ Raman study of UCAMM samples, and discuss the implications for their origin and significance.

\section{Experiments}

We analysed eight UCAMMs from the Concordia/CSNSM collection. Fragments of each UCAMM was micro-manipulated and transferred into a diamond compression cell. The fragments were then flattened to provide an optimal thickness for infrared spectroscopic transmission measurements. The infrared measurements were performed on the SMIS beam line at the synchrotron SOLEIL during several runs in the period from 2010 to 2016. The synchrotron beam was coupled to a Nicolet NicPlan infrared microscope. For the measurements presented in this analysis, the infrared spot size was optimized close to the diffraction limit, with $5 \times 5 \mu \mathrm{m}$ to $15 \times 15 \mu \mathrm{m}$ sampling windows, adapted to the geometry of each UCAMM fragment. A Thermo Fisher DXR spectrometer was used to record a Raman spectrum for each of the samples, using a laser source at $532 \mathrm{~nm}$ at minimum power (100 $\mu \mathrm{W}$ on sample) to prevent sample alteration. Ten scans with integration times of $20 \mathrm{~s}$ each were co-added for each spectrum. The spot size of the Raman spectra is lower than that of the IR spectra, of the order of a micron.

For five of the UCAMMS, light element analysis (C, N, O) was conducted at Université Paris 6 Jussieu using a Cameca SX100 or SXFive electron microprobe at $10 \mathrm{keV}$ and $40 \mathrm{nA}$ or $20 \mathrm{nA}$. Before analysis, the samples and standards were coated with a $9 \mathrm{~nm}$ layer of gold using a sputter-coating technique equipped with a quartz thickness monitoring. Care was taken to gold-coat the sample and the standard mounts at the same time whenever possible. The $\mathrm{C}, \mathrm{N}$, and $\mathrm{O} \mathrm{K} \alpha$ emission lines were recorded using a PC1 crystal (W-Si multilayer crystal), and were calibrated using graphite for $\mathrm{C}, \mathrm{BN}$ for $\mathrm{N}$, and $\mathrm{Fe}_{2} \mathrm{O}_{3}$ for $\mathrm{O}$. As UCAMMs contain variable proportions of minerals intimately mixed with the organic matter; the $\mathrm{Na}, \mathrm{Mg}, \mathrm{Al}, \mathrm{Si}, \mathrm{P}, \mathrm{S}, \mathrm{K}, \mathrm{Ca}$,
$\mathrm{Ti}, \mathrm{Cr}, \mathrm{Mn}$, and $\mathrm{Fe} \mathrm{K} \alpha$ lines were recorded as well, using adequate analysing crystals (LTAP, LPET, or LLIF) and calibrated on usual mineral standards (albite for $\mathrm{Na}$; Olivine for $\mathrm{Si}$ and $\mathrm{Mg}$; orthoclase for $\mathrm{Al}$ and $\mathrm{K}$; apatite for $\mathrm{P}$ and $\mathrm{Ca}$; pyrophanite (Mn$\mathrm{TiO} 3$ ) for $\mathrm{Mn}$ and $\mathrm{Ti}$; chromite $\left(\mathrm{Cr}_{2} \mathrm{O}_{3}\right)$ for $\mathrm{Cr}$; $\mathrm{Fe}_{2} \mathrm{O}_{3}$ for $\left.\mathrm{Fe}\right)$. Totals different from $100 \%$ can be due to porosity and matrix effects slightly differing between the standard and crushed samples or to a high concentration of an element not measured in the standard (e.g. Ni). Since these analyses were performed on crushed grains, only analyses with reasonable totals in wt $\%(85 \%<$ totals $<115 \%$ ) were selected to calculate the $\mathrm{N} / \mathrm{C}$ ratios. We note that the ratios between $\mathrm{C}, \mathrm{N}$, and $\mathrm{O}$ measured in a given sample are robust even in the cases of very anomalous totals as they are measured on the same detector. The typical statistical uncertainties for the determination of $\mathrm{C}, \mathrm{N}$, and $\mathrm{O}$ in the UCAMMs are on average $4 \mathrm{wt} \%, 1 \mathrm{wt} \%$, and $0.5 \mathrm{wt} \%$, respectively. The external uncertainty (reproducibility) on the $\mathrm{C}$ and $\mathrm{N}$ standards are on average $4 \mathrm{wt} \%$ and $2.5 \mathrm{wt} \%$. The intrinsic variability of a UCAMM sample N/C ratio is usually larger than the analytical uncertainty.

\section{Analysis}

The measured UCAMM infrared and Raman spectra are displayed in Fig. 2. The IR spectra are baseline corrected, in a similar way to previously reported in Dartois et al. (2013), and decomposed into individual contributions corresponding to specific vibrational modes. We also indicate the global fingerprint absorption band in the $1500-800 \mathrm{~cm}^{-1}$ spectral region, together with the silicates stretching complex contribution. We represent by different colours the various contributions to the spectra in Fig. 2. Except for the $\sim 1100-900 \mathrm{~cm}^{-1}$ spectral region, where silicate absorptions prevail if present, the spectra are dominated by the organic matter contribution. The broad band extending from about 3600 to $3000 \mathrm{~cm}^{-1}$ includes contributions from $\mathrm{OH}$ and $\mathrm{NH}$ stretching modes. The asymmetric profile of the band in this range, by comparison with laboratory analogues (Dartois et al. 2013), indicates a major contribution of 

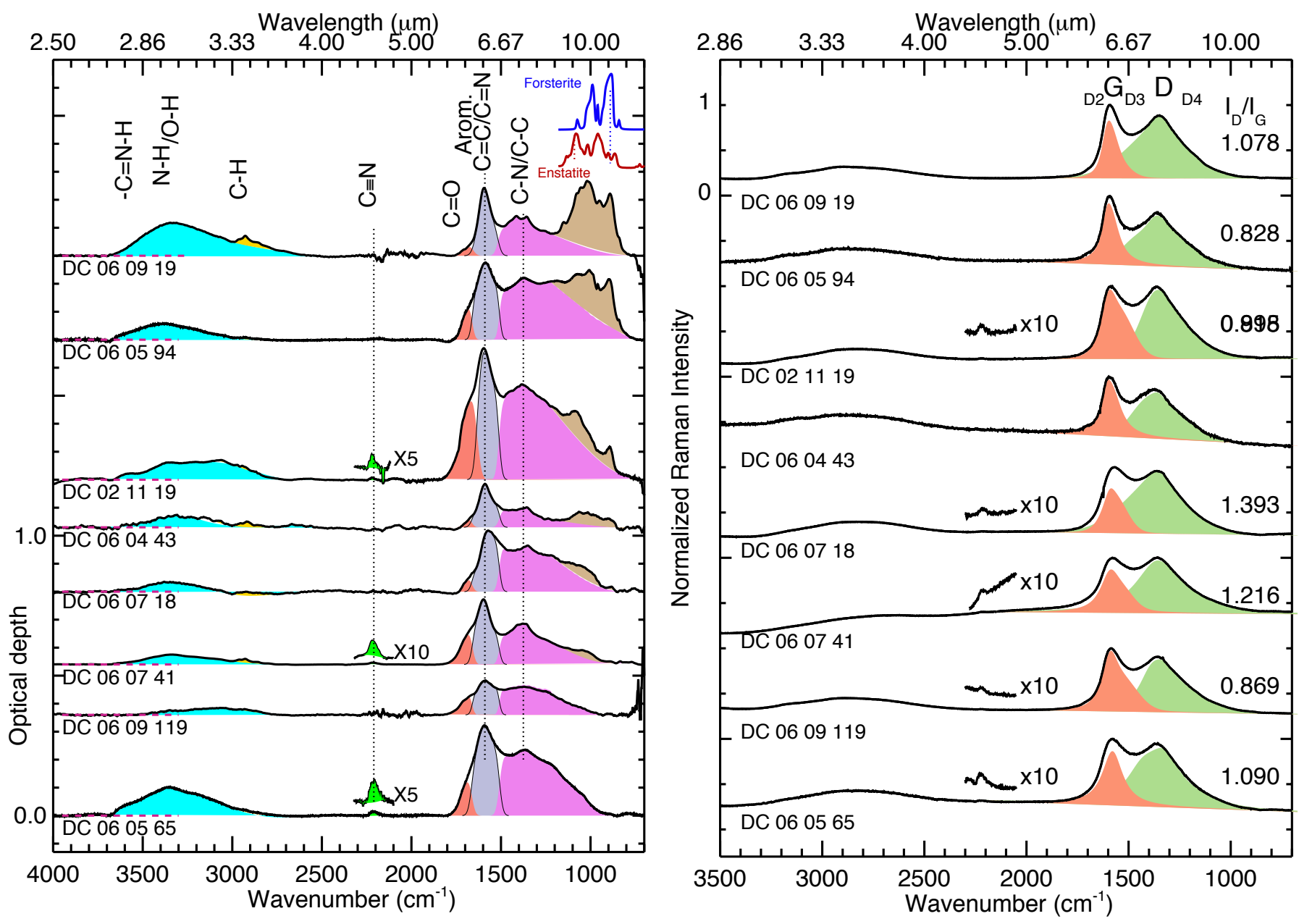

Fig. 2. Left: UCAMM samples $\mu$ FTIR optical depth spectra. The spectra have been vertically shifted for clarity (left horizontal dashed lines indicate the shift level). They are ordered from top to bottom by decreasing amount of silicate band absorption. The main band contributions are labelled above the upper spectrum. The spectra are deconvolved into several contributions shown with distinct colours, used in the analysis. A zoom on the nitrile region $\left(\sim 2200 \mathrm{~cm}^{-1}\right)$ is provided when a band was measured. Right: UCAMM samples Raman spectra, normalized to the $G$-band maximum. The spectra are analysed using a classical Raman bands fitting procedure (Sadezky et al. 2005; Kouketsu et al. 2014) contributing to the $D$ (green) and $G$ (red) bands. The $D / G$ band peak intensity ratio is given on the right, as defined in Busemann et al. (2007). A zoom on the nitrile region $\left(\sim 2200 \mathrm{~cm}^{-1}\right)$ is provided when a band was observed. Spectra are vertically shifted for clarity.

the $\mathrm{NH}$ stretching mode. We measured the amount of nitrogen in DC060594 and DC060565 independently (N/C ratios of 0.05 and 0.12 , locally exceeding 0.15 ; Dartois et al. 2013). The intrinsic intensity of the $\mathrm{OH}$ mode is about ten times higher than the $\mathrm{NH}$ stretching mode. It is probable that the broad and structureless feature between 3300 and $3500 \mathrm{~cm}^{-1}$ in DC060919 contains an $\mathrm{OH}$ component.

A few spectra possess a series of $\mathrm{CH}$ stretching mode absorptions, shown in yellow in the left panel of Fig. 2, with a close-up shown in Fig. 3, left panel. The main bands are due to aliphatic $\mathrm{CH}$ stretching modes $\left(\sim 2960 \mathrm{~cm}^{-1}\right.$, asymmetric $\mathrm{CH}_{3}$; $\sim 2920 \mathrm{~cm}^{-1}$, asymmetric $\mathrm{CH}_{2} ; \sim 2870-2850 \mathrm{~cm}^{-1}$, symmetric $\mathrm{CH}_{3}$ and $\mathrm{CH}_{2}$ ). A small contribution at $\sim 3050 \mathrm{~cm}^{-1}$ from the aromatic $\mathrm{CH}$ stretching mode is observed for the first time in UCAMMs. We also observed a potential small contribution of aldehyde $\mathrm{CH}$ at low wavenumber $\left(2820 \mathrm{~cm}^{-1}\right)$ in DC060919.

Three out of the eight UCAMM fragment analyses show an infrared $\mathrm{C} \equiv \mathrm{N}$ absorption feature at $2200 \mathrm{~cm}^{-1}$, which have also been measured with the Raman spectrometer for five UCAMMs (Fig. 3, centre panel) and previously observed by Dobrică et al. (2011) for DC060919. The main vibrational bands associated with the observed IR absorption and Raman emission bands are summarized in Table 1.

Unlike the other fragment, the DC021119 fragment was retrieved from a previous preparation where it was stuck in a carbon-tape for SEM analysis. The DC021119 UCAMM infrared spectrum is therefore affected, mainly in the carbonyl spectral region, and the carbonyl absorption for this UCAMM will thus not be considered in the following analysis.

The baseline corrected silicate optical depth contribution is shown in the right panel of Fig. 3, displayed together with several model absorption spectra of olivine and pyroxene magnesiumrich end members (forsterite and enstatite). The models were calculated with the continuous distribution of ellipsoids (CDE), spheroids (CDS), and hollow spheres (HS) to take into account the variabilities expected due to shape effects in scattering and absorption by randomly oriented particles that are small compared to the wavelength (Min et al. 2003). This statistical approach allows us to compare the amplitude of deformation induced by shapes in the spectra recorded and to infer the silicates composition (one pyroxene and two olivine bands are indicated by vertical dotted lines). It shows notably that the pyroxene 

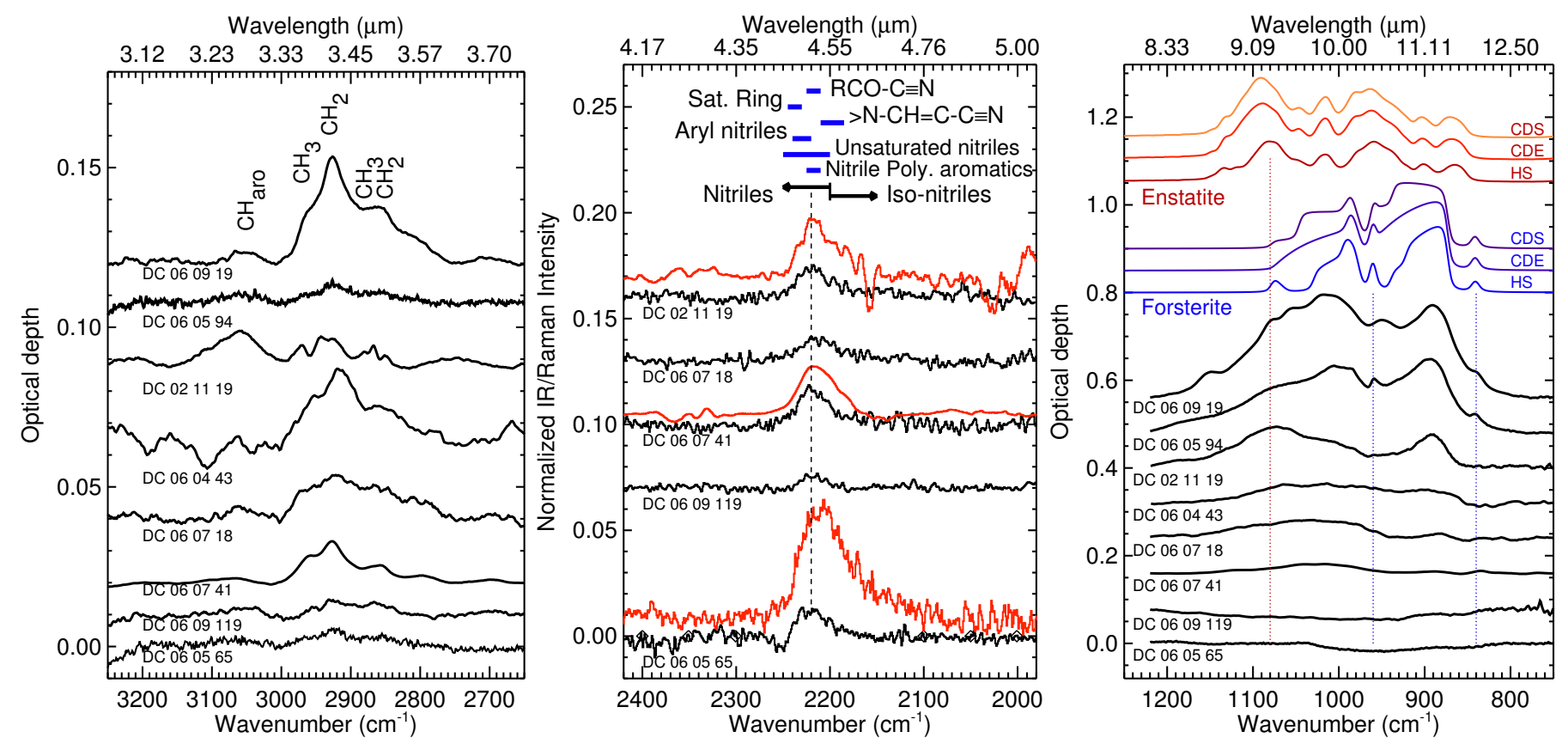

Fig. 3. Measured UCAMMs spectra. Left: CH stretching mode infrared optical depth. Centre panel: $\mu$ FTIR (red) and Raman (black) close-up spectra in the nitriles region, normalized to the $\mathrm{C}=\mathrm{C}(\mathrm{IR})$ and $G$ band (Raman) measured intensities. Right: baseline corrected silicate infrared optical depth, along with different absorption models for several size distributions of olivine (forsterite) and pyroxene (enstatite) magnesium rich end members (see text for details).

Table 1. Infrared and Raman band list.

\begin{tabular}{l|lc}
\hline \hline $\begin{array}{l}\text { Position } \\
\left(\mathrm{cm}^{-1}\right)\end{array}$ & \multicolumn{2}{|c}{ Mode } \\
\hline $3600-3000$ & $\mathrm{IR} / \mathrm{NH}$ stretch (br) & \\
3050 & $\mathrm{CH}$ aro. stretch & \\
2960 & $\mathrm{CH}_{3}$ asym. stretch & \\
2920 & $\mathrm{CH}_{2}$ asym. stretch & \\
2870 & $\mathrm{CH}_{3}$ sym. stretch & \\
2860 & $\mathrm{CH}_{2}$ sym. stretch & \\
2220 & $\mathrm{C} \equiv \mathrm{N}$ & $\mathrm{C} \equiv \mathrm{N}$ \\
$1750-1650$ & $\mathrm{C}=\mathrm{O}$ & \\
$1600-1580$ & $\mathrm{C}=\mathrm{C} / \mathrm{C}=\mathrm{N}$ & $\mathrm{C} \mathrm{sp}{ }^{2}$ \\
1475 & $\mathrm{CH} 2,3$ deformation & \\
$1400-1100$ & $\mathrm{C}-\mathrm{N} / \mathrm{C}-\mathrm{C}$ (br) & \\
1375 & $\mathrm{CH}{ }_{2,3}$ deformation & \\
$1370-1340$ & \multicolumn{2}{c}{$\mathrm{C} \mathrm{sp}^{2}$ “Defect" band } \\
$1100-800$ & $\mathrm{SiO}$ stretch (Silicates, br) \\
\hline
\end{tabular}

Notes. br: broad.

band complex still absorbs significantly at higher wavenumbers (above about 1070-1080 $\mathrm{cm}^{-1}$ ) than the olivine band. UCAMMs contain a mixture of silicates on a much smaller scale than that probed by classical IR wavelength microscopy. DC060919 and DC060594 display typical forsterite-like absorption bands, whereas DC021119 absorbs significantly above $1070 \mathrm{~cm}^{-1}$, indicating an enstatite-like contribution.

To provide a systematic comparison with the organic matter extracted from meteorite IOM, the published infrared spectra from Kebukawa et al. (2011), Orthous-Daunay et al. (2013), Quirico et al. (2014) were analysed in the exact same way and are discussed below. We also performed infrared measurements at the synchrotron Soleil SMIS beam line on IOM extracted from the Paris meteorite, kindly provided by V. Vinogradoff and
L. Remusat (IMPMC/ MNHN) ${ }^{1}$. The analysis of this spectrum was performed in the same way and added to the results displayed in Figs. 5 and 6. It is difficult to obtain IR spectra covering the full $4000-600 \mathrm{~cm}^{-1}$ range for the insoluble organic matter fraction of IDPs. A few exceptions with Hydrofluoric acid (HF) attack of IDPs to eliminate the inorganic fraction, followed by IR measurements, were recorded by e.g. Matrajt et al. (2005b), and are added for comparison to the analysis presented here.

The UCAMMs Raman spectra are analysed using a classical Raman bands fitting procedure decomposition contributing to the $D$ and $G$ bands, consisting of the deconvolution of five subbands (e.g. Sadezky et al. 2005; Kouketsu et al. 2014) grouped into two main contributions ("D" $=D_{1}+D_{2}+D_{4}$ and " $G$ " = $\left.D_{3}+G\right)$, shown in the right panel of Fig. 2 .

\section{Discussion}

The UCAMMs Raman spectra can be compared to previous measurements for meteoritic IOM (Busemann et al. 2007, see also Busemann et al. 2009, 2011; Alexander et al. 2017), Stardust (Rotundi et al. 2008, see also Sandford et al. 2006), and previous UCAMM measurements (Dobrică et al. 2011). The broad and relatively intense Raman $D$ band and intensity ratio of the $D$ and $G$ bands underscore the disordered nature of the carbonaceous network for UCAMMs organic matter. In the $D$-band full width at half maximum (FWHM) versus $D$ band position diagram and $D$-FWHM versus $G$-FWHM shown in Fig. 4, UCAMMs lie in the region associated with the most primitive meteorites and Stardust grains. Busemann et al. (2007) found and discussed a potential correlation of the atomic N/C element abundance ratios with the $D$ band FWHM (Fig. 8, Busemann et al. 2007) and a correlation of the atomic $\mathrm{H} / \mathrm{C}$ element abundance ratios with the $G$ band FWHM (Fig. 9, Busemann et al. 2007). The UCAMMs measured values are clearly not in line with what would be extrapolated from these

1 The full spectrum will be published by these authors. 

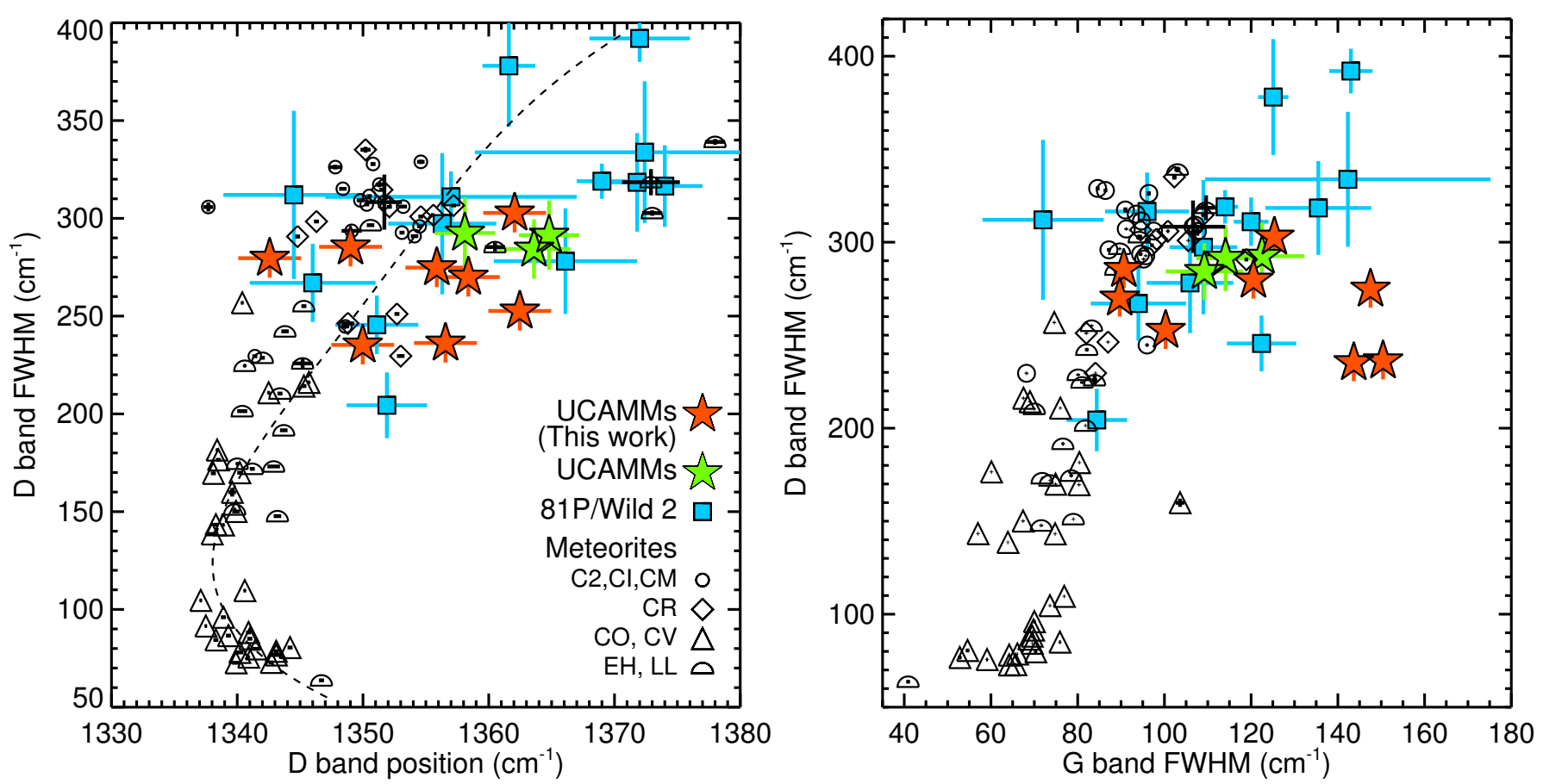

Fig. 4. Raman $D$ band full width at half maximum (FWHM) versus position (left) and $G$ band FWHM (right) diagrams, combining meteoritic IOM (C2, CI, CM: circles, CR: diamonds, triangles: CO, CV and EH, LL: half circles, Busemann et al. 2007), Stardust (squares, Rotundi et al. 2008), previous UCAMM measurements (green stars, Dobrică et al. 2011), and UCAMMs from this work (red stars). The fitted trend is associated with the organic matter primitivity; the most primitive meteorites have $D$ band width $\gtrsim 250 \mathrm{~cm}^{-1}$.

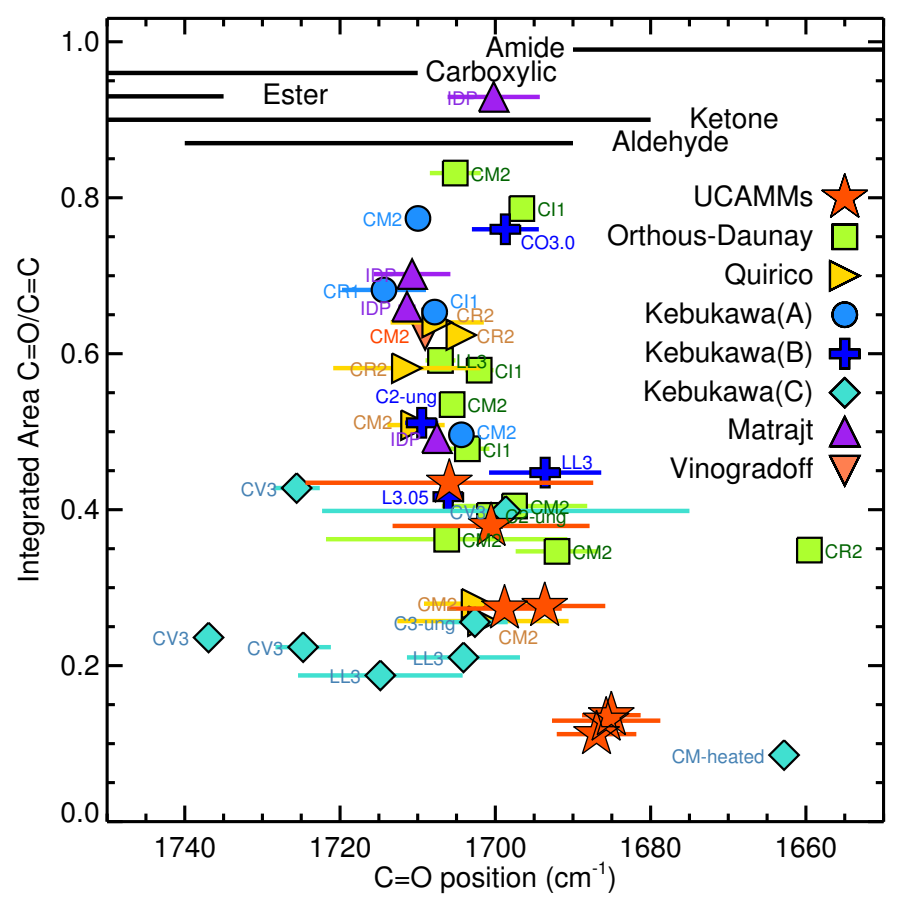

Fig. 5. Comparison of UCAMM infrared measurements for carbonyl, displayed with the corresponding infrared features in meteorites. Carbonyl-to-aromatic-carbon ratio as a function of the position of carbonyl stretching vibrations, and corresponding chemical functional groups.

correlations, showing that UCAMMs, with their high N/C and low $\mathrm{H} / \mathrm{C}$ abundances ratios, evolve following another trend.

The integrated optical depth of the carbonyl divided by the $\mathrm{C}=\mathrm{C}$ value is reported in Fig. 5 as a function of the carbonyl absorption central position. A chemical function information arises from the position of the carbonyl. Because the electrophile behaviour of neighbouring atoms has an influence on the bond and thus can alter its position in the spectrum, it is not possible to infer an exact attribution of the carbonyl by comparison with the expected classical positions. However, as they appear in the $1680-1705 \mathrm{~cm}^{-1}$ range, it seems to favour an aldehyde or ketone carbonyl function over carboxylic acids and ester. They fall close to the positions of the primitive classes A and B from the spectra from Kebukawa et al. (2011), Orthous-Daunay et al. (2013), and Quirico et al. (2014).

The integrated optical depth of the carbonyl absorption band divided by the $\mathrm{C}=\mathrm{C}$ value is reported in Fig. 6 (left panel) as a function of the integrated optical depth of the $\mathrm{CH}$ stretching mode to $\mathrm{C}=\mathrm{C}$ ratio. We did not include DC021119 in this plot because the tape contamination induces a spectral overlapping contamination in the $\mathrm{C}=\mathrm{O}$ region. The UCAMMs position in this diagram is specific as they clearly lie in the lower left corner with respect to the IOM from meteorites and IDPs. The low ratio of $\mathrm{C}=\mathrm{O}$ to $\mathrm{C}=\mathrm{C}$ of the UCAMMs confirms that the $\mathrm{O} / \mathrm{C}$ ratio in the organic matter of UCAMMs is lower than that of CR and $\mathrm{CM}$ meteorites and IDPs.

The low $\mathrm{CH}$ to $\mathrm{C}=\mathrm{C}$ ratio Fig. 6 (right panel) also indicates a low hydrogen content. In this diagram, UCAMMs for which atomic $\mathrm{H} / \mathrm{C}$ ratio were not available are set at a zero $\mathrm{H} / \mathrm{C}$ value. However, given the general trend observed with the measured infrared $\mathrm{CH} / \mathrm{C}=\mathrm{C}$ band ratio, these UCAMMs most probably are below $\mathrm{H} / \mathrm{C} \lesssim 0.5$. This value is further confirmed for the two UCAMMs for which a $\mathrm{H} / \mathrm{C}$ ratio was measured with the NanoSIMS (Duprat et al. 2010). The H/C in UCAMMs is substantially lower than those measured for meteorites from the infrared spectra or independently by other means by Alexander et al. (2007).

The physicochemical composition modifications undergone by meteorites, IDPs, and micrometeorites during their atmospheric entry is a long-standing question (e.g. 

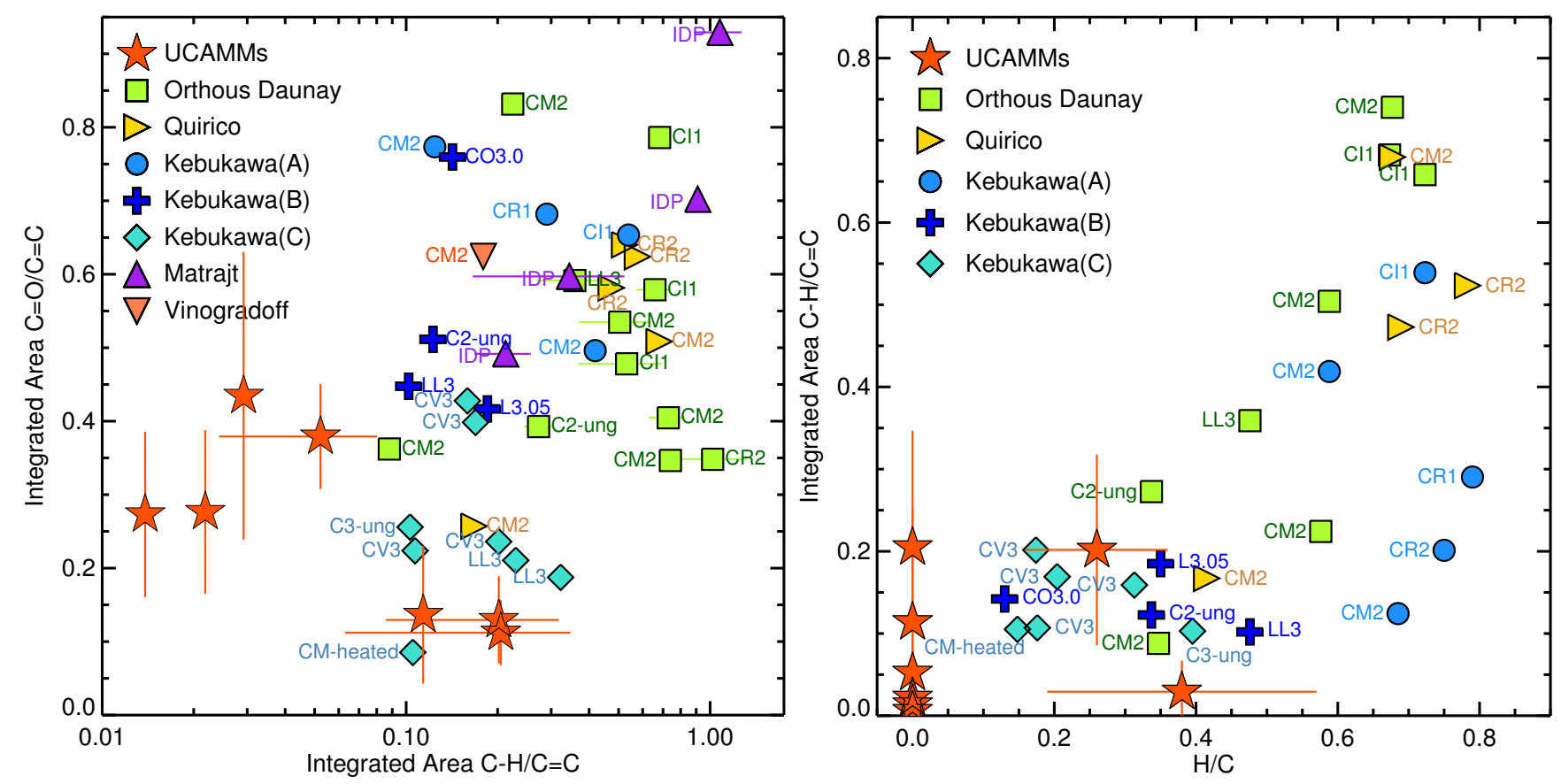

Fig. 6. Comparison of UCAMM infrared measurements for carbonyl, aromatic carbon, and hydrogen bonded to carbon, displayed with the corresponding infrared features in meteorite (Orthous-Daunay et al. 2013; Quirico et al. 2014; Kebukawa et al. 2011, separating the first three infrared subclasses defined in the Kebukawa spectral analysis) and IDP (Matrajt et al. 2005b) insoluble organic matter. Left: carbonyl-to-aromaticcarbon ratio as a function of the $\mathrm{CH}$ to $\mathrm{C}=\mathrm{C}$ areas; right: integrated optical depth area of the $\mathrm{CH}$ versus $\mathrm{C}=\mathrm{C}$ bands as a function of $\mathrm{H} / \mathrm{C}$ ion probe (NanoSIMS) independent measurements. The UCAMMs without available $\mathrm{H} / \mathrm{C}$ measurements are set at a zero $\mathrm{H} / \mathrm{C}$ value. Labels correspond to meteorite groups, as reported in these articles

Greshake et al. 1998; Toppani et al. 2001; Matrajt et al. 2005b; Suttle et al. 2017). In the Concordia micrometeorite collection, a large fraction $(\sim 35 \%)$ of the particles exhibit textures suggesting that they were not substantially altered during atmospheric entry (e.g. Dobrica et al. 2010). Several indicators point toward a moderate heating for most UCAMMs. Chemical and structural modifications expected on minerals during thermal processing give a strong upper limit on the temperature suffered by the UCAMMs. First of all, there is no significant magnetite conversion from pyrrhotite in UCAMMs, estimated to occur in the 500-900 ${ }^{\circ} \mathrm{C}$ range (e.g. Rietmeijer 2004; Craig 1974). The annealing of tracks in the minerals occurs at a temperature below $600{ }^{\circ} \mathrm{C}$. Some of the minerals in UCAMMs display these tracks (Charon et al. 2017). The high organic content of UCAMMs is also an indicator of at most moderate heating. The UCAMM organic matter Raman $G$ and $D$ band FWHMs are large; for some UCAMMs they lie above $140 \mathrm{~cm}^{-1}$ for the $G$ band, the largest among extraterrestrial organic matter, which points toward a low atmospheric entry heating by comparison to experimental simulations (e.g. Bonnet et al. 2015). The high amount of nitrogen in UCAMMs organic matter and the presence of $\mathrm{NH}$ stretching modes confirm the low heating; above about $500{ }^{\circ} \mathrm{C}$ these $\mathrm{NH}$ bonds should be reduced drastically, and this implies that the $\mathrm{N} / \mathrm{C}$ would otherwise be even higher before entry. The greatest unknown is probably the initial C-H content in UCAMMs, which may be reduced during atmospheric entry. IDPs often preserve higher aliphatic C-H content in their organic matter than measured for UCAMMs. Because IDPs are usually smaller in size, they may have suffered less heating during the atmospheric entry (e.g. Love \& Brownlee 1991). Undoubtedly ices, including nitrogen ices, are lost long before the atmospheric entry, in the evolving parent body, and during the journey of the dust grains toward the inner solar system. This evolution most probably helped to form their original macromolecular organic content as discussed in the UCAMMs formation scenarios (Dartois et al. 2013; Augé et al. 2016). The nitrogen budget of UCAMMs before atmospheric entry may be higher than measured in this study if they suffered some heating during atmospheric entry. The nitrogen-to-carbon atomic ratio is indeed expected to decrease with the heating of the particles (e.g. Bonnet et al. 2015), but should remain relatively stable up to about $500{ }^{\circ} \mathrm{C}$. Above such temperatures, the polyaromatic network is also strongly modified and the nitrile infrared signature disappears, whereas it is observed in some of the UCAMMs in this study. If the particles were heated to much higher temperatures, the measured $\mathrm{N} / \mathrm{C}$ ratios should be considered as lower limits of the initial N/C ratios as heating would result in nitrogen loss. The chemical element the most prone to substantial loss from the organic content of UCAMMs is hydrogen as it is the most labile. The CH content in UCAMMs is variable, as measured in the infrared spectra, and in some cases relatively low. It is therefore not possible to definitely rule on the pre-atmospheric entry $\mathrm{CH}$ content of UCAMMs. It may be higher before atmospheric entry than measured in this study and reduced upon atmospheric entry. It could also be the initial and preserved $\mathrm{CH}$ content, with the hydrogen being mainly bound as $\mathrm{NH}$, as currently observed. However, taken together, the above-mentioned arguments and measurements provide a substantial body of evidence that UCAMMs probably did not suffer a temperature of more than about $100-500{ }^{\circ} \mathrm{C}$ during atmospheric entry.

\subsection{Nitrogen contribution and the N/C solar system gradient}

The position of the $\mathrm{C} \equiv \mathrm{N}$ band measured in the IR and in the Raman spectra, shown in the central panel of Fig. 3, corresponds to a nitrile function; an isonitrile group would absorb at lower frequencies (e.g. Bonnet et al. 2015). The absorption profile 
E. Dartois et al.: Dome C ultracarbonaceous Antarctic micrometeorites
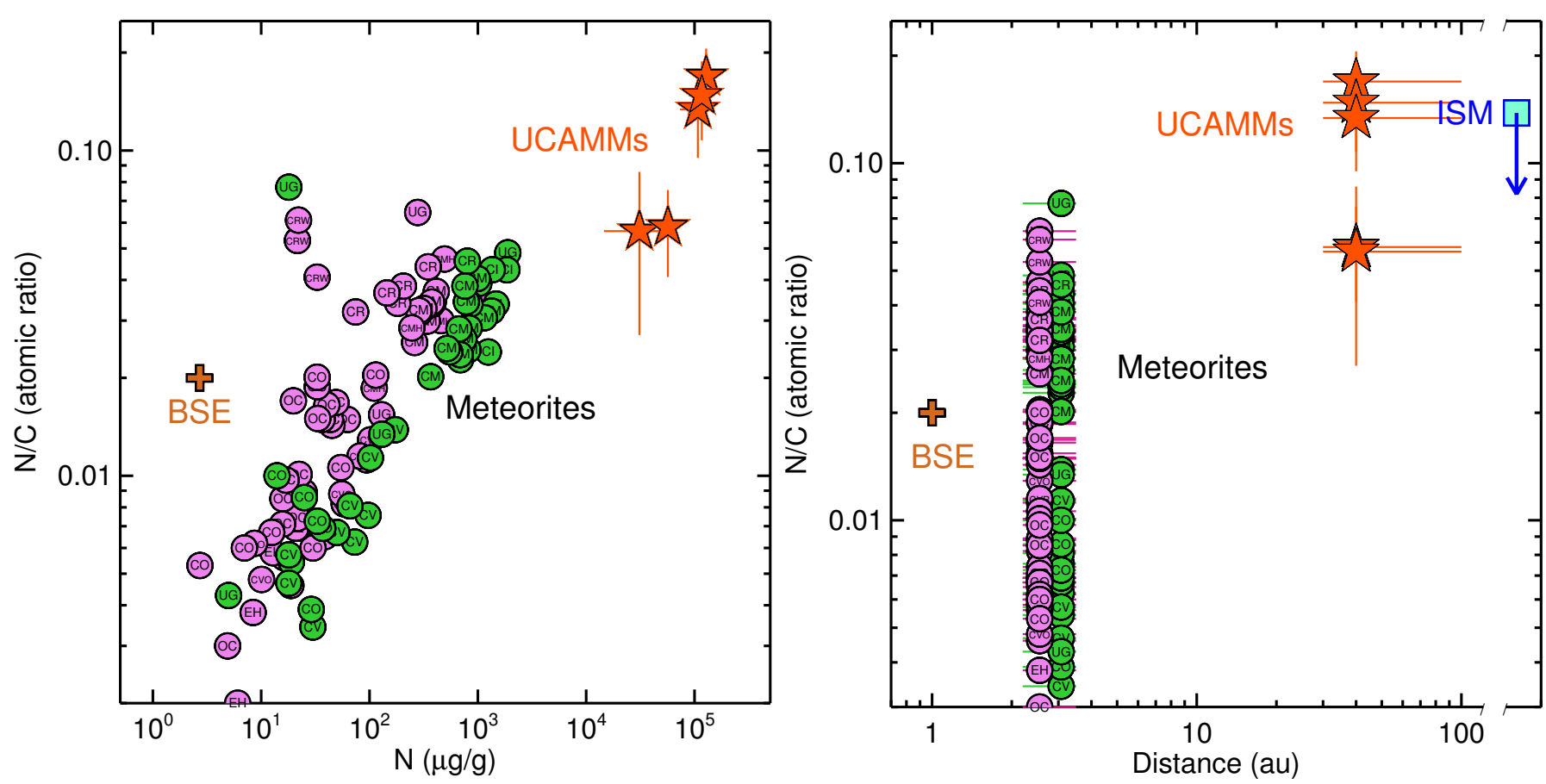

Fig. 7. UCAMM nitrogen abundances relative to carbon (atomic ratio) are compared to solar system solids: meteorites (Kerridge 1985; Alexander et al. 2007, green and purple circles, respectively; labels correspond to meteorites groups as reported in these articles), the Bulk Silicate Earth (BSE, Bergin et al. 2015), and the interstellar medium (see text for details). Left: reported in function of bulk nitrogen weight fraction; right: reported in function of heliocentric distance. Figure A.1 summarizes the distance, N/C, and $\mathrm{N}$ weight fraction information in a single plot. UCAMMs values are reported in Table B.1.

in the fingerprint region $\left(1500-800 \mathrm{~cm}^{-1}\right)$ is significantly different in UCAMMs compared to meteorites' IOM. It is particularly evident when the organic content is the highest and the silicates represent a negligible fraction of the absorption profile (lower spectra of left panel in Fig. 2). The absorption bands peak around $1380 \mathrm{~cm}^{-1}$, whereas in IOM spectra the maximum of the broad absorptions peaks around $1200 \mathrm{~cm}^{-1}$ (e.g. Fig. 1 Dartois et al. 2014; Quirico et al. 2014; Orthous-Daunay et al. 2013; Kebukawa et al. 2011), with variable methyl and methylene deformation mode contributions on top of the profile at higher wavenumbers. This UCAMM fingerprint region profile is reminiscent of the nitrogen-rich carbonaceous network, as observed in the spectra of laboratory a-CNH analogues (e.g. Augé et al. 2016; Quirico et al. 2008; Lazar et al. 2008; Gerakines et al. 2004; Fanchini et al. 2002; Rodil et al. 2001; Hammer et al. 2000; Ong 1996), in agreement with the higher nitrogen fraction in the organic phase of UCAMMs (Dobrică et al. 2011; Dartois et al. 2013). If the main nitrogen contribution to the UCAMMs is not the $\mathrm{C} \equiv \mathrm{N}$, but $\mathrm{C}=\mathrm{N}$ and $\mathrm{C}-\mathrm{N}$ bonds contributing to the infrared spectra at lower frequencies, what makes the $\mathrm{C} \equiv \mathrm{N}$ feature unique is that it falls in a clean spectral region, and when measured in organic matter, it is in most cases the sign of a high nitrogen content. UCAMMs are probably one of the poles in the nitrogen history of the solar system solids, probably sharing commonalities with some of the nitrogen-rich hot spots found at much smaller scales (i.e. lower organic matter fraction) in some IDPs (e.g. Aléon 2010). The nitrogen-to-carbon abundance ratio in UCAMMs, measured with an electron microprobe for five of them, are shown in Fig. 7 (left panel) together with their nitrogen absolute abundances (in weight fraction), and are compared to other solar system solids. The N/C is one of the indicators used to discriminate between different reservoirs for the organic matter in solar system solids. Although difficult to evaluate, the Bulk Silicate Earth (BSE) N/C value lies about an order of magnitude lower (Halliday 2013; Marty 2012; Bergin et al. 2015, and references therein). The interstellar medium N/C is somewhat more difficult to constrain as - in contrast to measurements on interplanetary carbonaceous dust nitrogen in diffuse ISM solids is spectroscopically elusive. Nevertheless, an upper limit can be determined assuming that the depletion of nitrogen and carbon observed in the diffuse medium is locked into carbonaceous dust. Considering the missing fraction of nitrogen $(\delta \mathrm{N})$ one can form the elemental ratio $\mathrm{N} / \mathrm{C} \lesssim$ $(\delta \mathrm{N} \times[\mathrm{N}]) /(\delta \mathrm{C} \times[\mathrm{C}]) \approx(0.2 \times 80 \mathrm{ppm}) /(0.4 \times 290 \mathrm{ppm}) \approx 0.14$ (e.g. Verstraete 2011, and references therein). This ratio represents a stringent upper limit for the N/C ISM value that is in agreement with the fact that spectroscopic observation of both the aromatic infrared bands emission carriers, also called "astrophysical PAHs" (polycyclic aromatic hydrocarbons), and hydrogenated amorphous carbon solids in the ISM do not show a large incorporation of nitrogen as an heteroatom. Abundances of N/C in the organic matter from measurements for meteorites from the asteroid belt (Kerridge 1985) are also shown in Fig. 7. Their N/C ratio and absolute $\mathrm{N}$ abundance are both lower than in UCAMMs, with a slight overlap for the extremes in each distribution. The bulk $\mathrm{N}$ abundance in meteorites is lower than that of UCAMM by up to an order of magnitude. UCAMMs are placed in a $\mathrm{N} / \mathrm{C}$ versus estimated heliocentric distance diagram in the right panel of Fig. 7. As discussed in Bergin et al. (2015), Millar (2015), and Lee et al. (2010), the N/C ratio is sensitive to the disc chemistry and the radial transport, with a greater retention of $\mathrm{C}$ over $\mathrm{N}$ for thermal or impact events. Disc models (e.g. Piso et al. 2016) endeavor to demonstrate to what extent the $\mathrm{C} / \mathrm{O}$ and $\mathrm{N} / \mathrm{C}$ ratio can be related to the abundance of specific carriers, the composition of the ice (dominated or not by water ice, which controls the binding energy of more volatile ices), and the effect on the temporal evolution of disc temperatures on the position of the snow lines of the volatile species. 

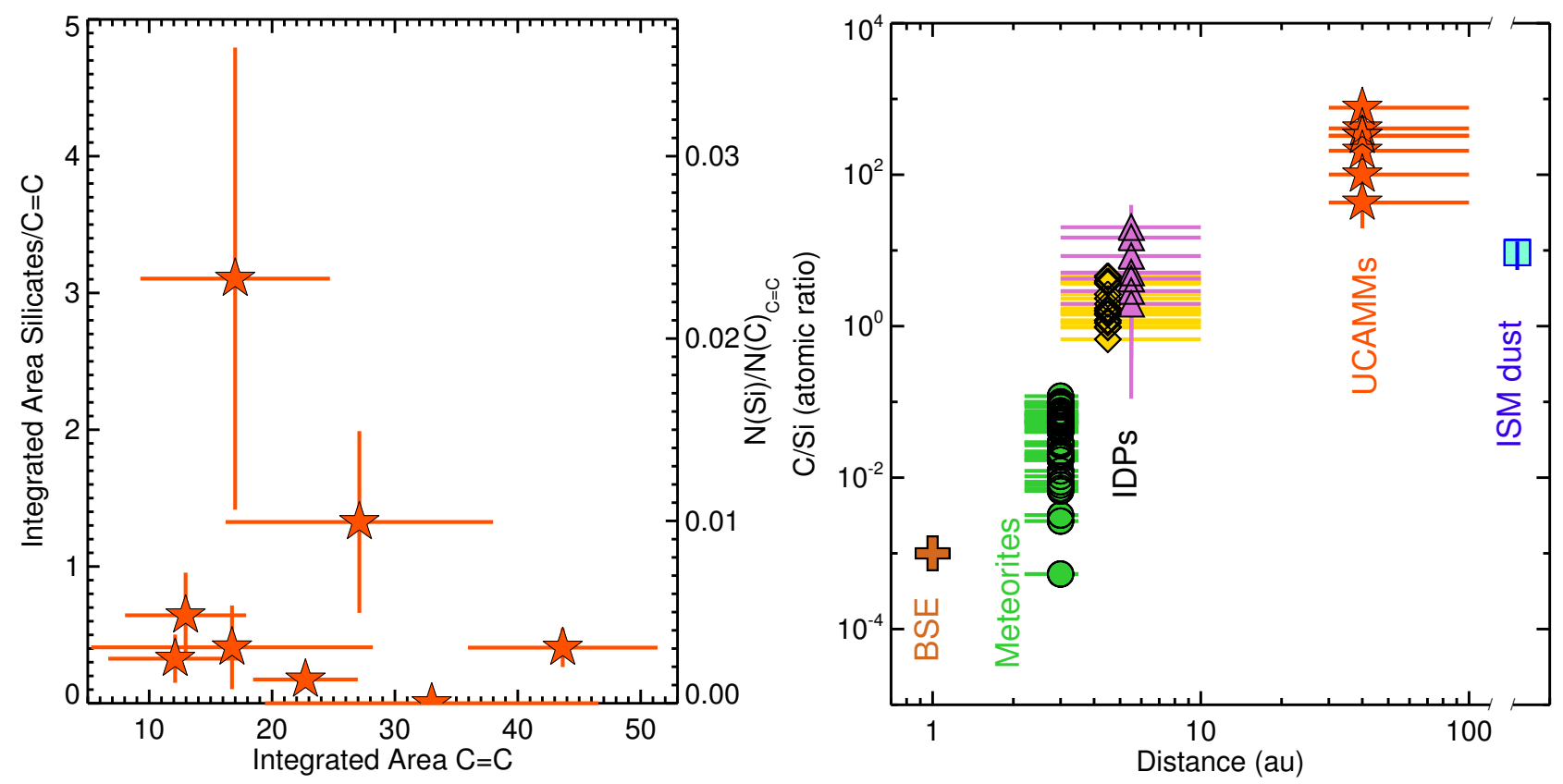

Fig. 8. Left: silicates-to-aromatic $\mathrm{C}=\mathrm{C}$ integrated area ratio and corresponding silicon-to-carbon atomic ratio (right axis, see text for details) in UCAMMs. Right: UCAMMs (stars) carbon abundances relative to silicon (atomic ratio) are compared to solar system solids: IDPs (Matrajt et al. 2005b; Thomas et al. 1993, triangles and diamonds, respectively), meteorites (Kerridge 1985, circles), the bulk silicate Earth (BSE, Bergin et al. 2015, cross), and the interstellar medium (square, see text for details).

Up to the planetesimals formation phase, observations show outer regions dense and cold enough to condense even the most volatile species. This includes the nitrogen molecule that is unfortunately weakly infrared active via ice interaction induced transitions, and thus escaping to date a direct abundance determination in ice mantles in this phase. These condensation phases are indirectly traced by the observation of related species in the gas phase, such as the $\mathrm{N}_{2} \mathrm{H}^{+}$radical.

Following this early phase, and for up to $4 \mathrm{Gyr}$, larger icy bodies at large heliocentric distances can retain volatiles such as $\mathrm{CH}_{4}$ and $\mathrm{N}_{2}$ on their surfaces. An emblematic object of this type is Pluto, with a detailed spatial record of $\mathrm{CH}_{4}$ - and $\mathrm{N}_{2}$-rich ices at its surface, recently mapped by the New Horizons mission (e.g. Protopapa et al. 2017). Different terrains and ice compositions were observed, and the dynamical history of Pluto seems to favour ices segregations, with regions stable for very long periods of time, possibly over the age of Pluto itself (Hamilton et al. 2016). Pluto is among the largest bodies with (sub)surface $\mathrm{CH}_{4}$ and $\mathrm{N}_{2}$ volatile ices; however, in the Kuiper belt and the Oort cloud regions many smaller icy bodies should have (sub)surface $\mathrm{N}_{2}$ and $\mathrm{CH}_{4}$ dominated ices (e.g. Brown et al. 2011). These surfaces are exposed to Galactic cosmic-ray irradiation, and macromolecular precursors can be synthesized by irradiation of such ices (see the scenarios and experiments detailed in Dartois et al. 2013; Augé et al. 2016). In UCAMMs we see little evidence of a pristine, directly incorporated and unmodified, interstellar dust organic component. If the UCAMM organic matter was produced closer to the Sun by an alternative scenario, it would be difficult to reconcile with the high $\mathrm{N} / \mathrm{C}$ and $\mathrm{D} / \mathrm{H}$ and lower $\mathrm{O} / \mathrm{C}$ ratios than found in meteorite IOM. The overall results indicate that the UCAMM bulk organic matter is not a direct heritage from and incorporation of the presolar cloud.

\subsection{The $\mathrm{C} / \mathrm{Si}$ solar system gradient}

The silicon-to-carbon abundance ratio in UCAMMs can be evaluated by forming the ratio of the integrated silicates band around $1000 \mathrm{~cm}^{-1}$ to the contribution from the $\mathrm{C}=\mathrm{C}$ band around $1590 \mathrm{~cm}^{-1}$, which largely dominates the carbon network. Using the integrated absorption cross sections of the silicates band of $1.8 \pm 0.2 \times 10^{-16} \mathrm{~cm} / \mathrm{Si}$ (Matrajt et al. 2005b; Bowey \& Adamson 2002; Dartois 1998) and that of the $C=C$ band of $1.5 \pm 0.5 \times 10^{-18} \mathrm{~cm} / \mathrm{C}$ evaluated in Dartois et al. (2013), this ratio can be converted into $\mathrm{Si} / \mathrm{C}$ values. They are reported in the right ordinate of Fig. 8, showing that the UCAMM silicates content is low $(\mathrm{Si} / \mathrm{C} \lesssim 0.03$ ). This $\mathrm{Si} / \mathrm{C}$ ratio measured in the infrared confirms the ultracarbonaceous nature of the fragments analysed. In this figure are displayed IDPs measurements of the Si/C by Thomas et al. (1993) and values retrieved from the infrared spectra from Matrajt et al. (2005b). For the latter, the $\mathrm{C} / \mathrm{Si}$ value reported for each of the seven IDPs analysed, is the mean of the C/Si given in Table 4 of Matrajt et al. (2005b), including only aliphatic carbons, and a reevaluation based on the infrared spectra of the maximum $\mathrm{C}$ possibly hidden in the $\mathrm{C}=\mathrm{C}$ band absorption region. The vertical lines indicate the full range between the two calculated values. The major flux of such IDPs is attributed to parent bodies coming from Jupiter-family comets and asteroids, with heliocentric distances in the 3-10 a.u. range (Poppe et al. 2011; Poppe 2016). Measurements for meteorites from the asteroid belt (Kerridge 1985) are also shown. The bulk silicate Earth (BSE, Bergin et al. 2015, and references therein) $\mathrm{C} / \mathrm{Si}$ value lies orders of magnitude below. The $\mathrm{C} / \mathrm{Si}$ can also be evaluated from interstellar medium measurements. An upper limit can be determined by simply forming the cosmic abundance ratio for carbon and silicon, which is around 10, based on recent measurements (Jenkins 2014, 2009; Jenkins \& Tripp 2011; Przybilla et al. 2013). The lower bound can be estimated for carbon by looking either at the depletions, attributed mainly to solids formation, or also by quantifying the spectroscopically identified carbonaceous dust (e.g. Dartois et al. 2015, and references therein) or the carbon fraction required in dust models (Jones 2016; Draine 2015; Wang et al. 2014). Most of the silicon is locked into the formation of inorganic compounds (mainly amorphous silicates in the diffuse interstellar medium; 
Kemper et al. 2004). The lower bound is therefore around $40 \%$ of the cosmic abundance. We report the $\mathrm{C} / \mathrm{Si}$ ratio as a function of the estimated heliocentric distance for the different objects considered (Fig. 8, right panel), and compare it to the interstellar medium. The UCAMMs display the highest ratios above the meteorites and, importantly also above the interstellar value, which is considered the maximum direct $\mathrm{C} / \mathrm{Si}$ abundance heritage value. As stated in Millar (2015), the carbon abundance appears to be closer to normal value (i.e. $\mathrm{C} / \mathrm{Si}$ cosmic abundance) in the cooler materials that reside in the outer solar system, but globally the incorporation of carbon into refractory bodies is difficult. The preferential destruction of carbon grains over silicates in the inner protosolar disc (Lee et al. 2010) would naturally explain a carbon deficiency gradient.

Recent models propose that a large fraction of carbon in the early solar system was removed from the dust component, especially in the inner regions of the solar system. These models thus predict a radial dependence of the abundance of carbon dust and could explain the depletion observed for the carbon abundance in planetesimals in the asteroid belt and in the terrestrial planet region (e.g. Gail \& Trieloff 2017). These variations may have observational consequences for protoplanetary discs. In the remote observations of protoplanetary discs, PAH emission lines and carbonaceous dust carriers in general, are conspicuous by their absence, whereas silicates are clearly observed in emission in the zones approximately corresponding to the sizes of the actual inner solar system. In addition to the inherent emissivity lower contrast in bands and/or lines for many carbonaceous solids compared to silicates, such a $\mathrm{C} / \mathrm{Si}$ radial variation could play an important role in the difficulty encountered to detect them.

Finding a high $\mathrm{C} / \mathrm{Si}$ value in an extraterrestrial dust grain as is found in UCAMMs (C/Si $\gtrsim 10)$ and some IDPs - associated with a high N/C ratio (Duprat et al. 2010; Dartois et al. 2013), can thus most probably be assigned to an incorporation via a mechanism occurring in the outer solar nebula, such as the proposed irradiation scenario for UCAMMs; this is suggested by the $\mathrm{C} / \mathrm{Si}$ gradient and also because the direct incorporation from the diffuse ISM would apparently lead to a lower C/Si value.

\section{Conclusion}

Infrared and Raman $\mu$ spectroscopy spectra of eight UCAMMs fragments provide a more comprehensive picture on the physicochemical composition of UCAMMs. The UCAMMs clearly contain a large amount, usually well over a micron in size, of $\mathrm{N}$-rich organic matter that is not found in other types of extraterrestrial matter. The high carbon abundance relative to silicon in UCAMMs is well above that of most solar system primitive samples (IDPs, meteorites). It is also, for some of them, significantly above the mere interstellar $\mathrm{C} / \mathrm{Si}$ value which is probably not compatible with the hypothesis of a direct incorporation of an interstellar precursor. This favours scenarios in which the $\mathrm{N}$ enrichment is acquired as a secondary physicochemical process occurring during the protoplanetary phase or later, and not a heritage from pristine incorporated interstellar matter. The UCAMMs micrometeorites reveal the existence of nitrogenrich precursors formed beyond the nitrogen snow line, and a chemistry regime occurring in the solar system in outer regions. UCAMMs are additional evidence for the presence of a positive gradient of the $\mathrm{C} / \mathrm{Si}$ and $\mathrm{N} / \mathrm{C}$ abundance ratios with heliocentric distance, as expected from protoplanetary disc evolution models and progressively revealed by astrophysical disc observations.
Acknowledgements. We acknowledge SOLEIL for the use of the synchrotron radiation facilities. The authors warmly thank Paul Dumas for the discussions and support over many years and for the successful realization of these experiments. The authors are grateful to Bruno Crane, Nicolas Szwec, and Silvin Hervé for their help in the mechanical designs used to perform the experiments. We acknowledge Y. Kebukawa for kindly providing us with her original data. The authors would like to cordially thank Martin David Suttle for his constructive comments. Part of the equipment used in this work has been financed by the French INSU-CNRS program "Physique et Chimie du Milieu Interstellaire" (PCMI). The measurements of the N/C with the electron microprobe were performed thanks to the CAMPARIS team at Jussieu. This work was supported by the ANR projects COSMISME (Grant ANR- 2010-BLAN-0502) and OGRESSE (Grant ANR2011-BS56-026-01) of the French Agence Nationale de la Recherche as well as INSU, IN2P3, CNES, DIM-ACAV (Région Ile de France), CNRS, and Université Paris-Sud. This work is part of the JWST emblematic project from the LABEX-P2IO. We are also grateful to the French and Italian polar institutes IPEV and PNRA, for their financial and logistic support of the micrometeorites Concordia collection.

\section{References}

Aléon, J. 2010, ApJ, 722, 1342

Alexander, C. M. O., Fogel, M., Yabuta, H., \& Cody, G. D. 2007, Geochim. Cosmochim. Acta, 71, 4380

Alexander, C. M. O., Cody, G. D., De Gregorio, B. T., Nittler, L. R., \& Stroud, R. M. 2017, Chemie der Erde/Geochemistry, 77, 227

Augé, B., Dartois, E., Engrand, C., et al. 2016, A\&A, 592, A99

Bardin, N., Slodzian, G., Wu, T.-D., et al. 2014, Lun. Planet. Sci. Conf., 45, 2647 Bergin, E. A., Blake, G. A., Ciesla, F., Hirschmann, M. M., \& Li, J. 2015, Proc. Nat. Acad. Sci., 112, 8965

Bonnet, J.-Y., Quirico, E., Buch, A., et al. 2015, Icarus, 250, 53

Bowey, J. E., \& Adamson, A. J. 2002, MNRAS, 334, 94

Brown, M. E., Burgasser, A. J., \& Fraser, W. C. 2011, ApJ, 738, L26

Busemann, H., Alexander, M. O., \& Nittler, L. R. 2007, Meteor. Planet. Sci., 42, 1387

Busemann, H., Nguyen, A. N., Cody, G. D., et al. 2009, Earth Plan. Sci. Lett., 288,44

Busemann, H., Spring, N. H., Alexander, C. M. O., \& Nittler, L. R. 2011, Spectr. Lett., 44, 554

Charon, E., Engrand, C., Benzerara, K., et al. 2017, Lun. Planet. Sci. Conf., 48, 2085

Craig 1974, Survey of data sources on sulfide phase equilibria. In Sulfide Mineralogy, ed. P. H. Ribbe, CS 3-23, Reviews in Mineralogy I, Mineralogical Society of America

Dartois, E. 1998, Ph.D. Thesis

Dartois, E., Engrand, C., Brunetto, R., et al. 2013, Icarus, 224, 243

Dartois, E., Geballe, T. R., Pino, T., et al. 2014, Geochem. J., 48, 511

Dartois, E., Alata, I., Engrand, C., et al. 2015, Bull. Soc. Roy. Sci. de Liège, 84, 7

Dobrica, E., Engrand, C., Duprat, J., \& Gounelle, M. 2010, Meteor. Planet. Sci. Suppl., 73, 5213

Dobrică, E., Engrand, C., Quirico, E., Montagnac, G., \& Duprat, J. 2011, Meteor. Planet. Sci., 46, 1363

Draine, B. T. 2015, IAU General Assembly, 22, 2253136

Duprat, J., Engrand, C., Maurette, M., et al. 2006, Meteor. Planet. Sci. Suppl., 41, 5239

Duprat, J., Engrand, C., Maurette, M., et al. 2007, Adv. Space Res. 39, 605

Duprat, J., Dobrică, E., Engrand, C., et al. 2010, Science, 328, 742

Engrand, C., Benzerara, K., Leroux, H., et al. 2015, Lun. Planet. Sci. Conf., 46, 1902

Engrand, C., Duprat, J., Dartois, E., Godard, M., \& Delauche, L. 2017, EGU General Assembly Conference Abstracts, 19, 9979

Fanchini, G., Tagliaferro, A., Conway, N. M., \& Godet, C. 2002, Phys. Rev. B, 66,195415

Gail, H.-P., \& Trieloff, M. 2017, A\&A, 606, A16

Gerakines, P. A., Moore, M. H., \& Hudson, R. L. 2004, Icarus, 170, 202

Greshake, A., Kloeck, W., Arndt, P., et al. 1998, Meteor. Planet. Sci., 33, 267

Halliday, A. N. 2013, Geochim. Cosmochim. Acta, 105, 146

Hamilton, D. P., Stern, S. A., Moore, J. M., \& Young, L. A. 2016, Nature, 540, 97

Hammer, P., Lacerda, R. G., Droppa, R., Jr., \& Alvarez, F. 2000, Diamond and Related Materials, 9, 577

Janches, D., Heinselman, C. J., Chau, J. L., Chandran, A., \& Woodman, R. 2006,

J. Geophys. Res. (Space Physics), 111, A07317

Jenkins, E. B. 2009, ApJ, 700, 1299

Jenkins, E. B. 2014, ArXiv e-prints [arXiv: 1402 .4765] 
Jenkins, E. B., \& Tripp, T. M. 2011, ApJ, 734, 65

Jones, A. 2016, IAU Proc., IAU XXIX General Assembly, 29A, 313

Kebukawa, Y., Alexander, C. M. O., \& Cody, G. D. 2011, Geochim. Cosmochim Acta, 75, 3530

Kemper, F., Vriend, W. J., \& Tielens, A. G. G. M. 2004, ApJ, 609, 826

Kerridge, J. F. 1985, Geochim. Cosmochim. Acta, 49, 1707

Kouketsu, Y. 2014. Island Arc, 23, 33

Lazar, G., Bouchet-Fabre, B., Zellama, K., et al. 2008, J. Appl. Phys., 104, 073534

Lee, J.-E., Bergin, E. A., \& Nomura, H. 2010, ApJ, 710, L21

Love, S. G., \& Brownlee, D. E. 1991, Icarus, 89, 26

Love, S. G., \& Brownlee, D. E. 1993, Science, 262, 550

Marty, B. 2012, Earth Plan. Sci. Lett., 313, 56

Matrajt, G., Muñoz Caro, G. M., Dartois, E., et al. 2005a, A\&A, 433, 979

Matrajt, G., Brownlee, D. E., Joswiak, D. J., \& Taylor, S. 2005b, 36th Annual Lun. Planet. Sci. Conf., 36

Millar, T. J. 2015, Plasma Sources Science Technology, 24, 043001

Min, M., Hovenier, J. W., \& de Koter, A. 2003, A\&A, 404, 35

Nakamura, T., Noguchi, T., Ozono, Y., Osawa, T., \& Nagao, K. 2005, Meteor Planet. Sci. Suppl., 40, 5046

Nesvorný, D., Jenniskens, P., Levison, H. F., et al. 2010, ApJ, 713, 816

Nesvorný, D., Vokrouhlický, D., Pokorný, P., \& Janches, D. 2011, ApJ, 743, 37

Ong, C. 1996, Thin Solid Films, 280, 1

Orthous-Daunay, F.-R., Quirico, E., Beck, P., Brissaud, O., Dartois, E., Pino, T., \& Schmitt, B. 2013, Icarus, 223, 534

Piso, A.-M. A., Pegues, J., \& Öberg, K. I. 2016, ApJ, 833, 203

Poppe, A. R. 2016, Icarus, 264, 369

Poppe, A., James, D., \& Horányi, M. 2011, Planet. Space Sci., 59, 319

Prasad, M. S., Rudraswami, N. G., \& Panda, D. K. 2013, J. Geophys. Res. (Planets), 118, 2381

Protopapa, S., Grundy, W. M., Reuter, D. C., et al. 2017, Icarus, 287, 218
Przybilla, N., Nieva, M. F., Firnstein, M., \& Butler, K. 2013, EAS Pub. Ser., 64, 37

Quirico, E., Montagnac, G., Lees, V., et al. 2008, Icarus, 198, 218

Quirico, E., Orthous-Daunay, F.-R., Beck, P., et al. 2014, Geochim. Cosmochim. Acta, 136, 80

Rietmeijer, F. J. M. 2004, Meteoritics and Planetary Science, 39, 1869

Rodil, S. E., Ferrari, A. C., Robertson, J., \& Milne, W. I. 2001, J. Appl. Phys., 89,5425

Rotundi, A., Baratta, G. A., Borg, J., et al. 2008, Meteo. Planet. Sci., 43, 367

Sadezky, A., Muckenhuber, H., Grothe, H., Niessner, R., \& Poschl, U. 2005, Carbon, 43, 1731

Sandford, S. A., Aléon, J., Alexander, Conel, M. O. 'D., et al. 2006, Science, 314,1720

Suttle, M. D., Genge, M. J., Folco, L., \& Russell, S. S. 2017, Geochim. Cosmochim. Acta, 206, 112

Taylor, S., Lever, J. H., \& Harvey, R. P. 1996, Meteor. Planet. Sci. Suppl., 31,

Taylor, S., Alexander, C. M. O., \& Wengert, S. 2008, Lun. Planet. Sci. Conf., 39, 1628

Thomas, K. L., Blanford, G. E., Keller, L. P., Klock, W., \& McKay, D. S. 1993, Geochim. Cosmochim. Acta, 57, 1551

Toppani, A., Libourel, G., Engrand, C., \& Maurette, M. 2001, Meteor. Planet. Sci., 36, 1377

van Ginneken, M., Folco, L., Cordier, C., \& Rochette, P. 2012, Meteor. Planet. Sci., 47, 228

Verstraete, L. 2011, Eur. Phys. J. Web Conf., 18, 01001

Wang, S., Li, A., \& Jiang, B. W. 2014, Planet. Space Sci., 100, 32

Yabuta, H., Itoh, S., Noguchi, T., et al. 2012, Lun. Planet. Sci. Conf., 43, 2239

Yabuta, H., Noguchi, T., Itoh, S., et al. 2017, Geochim. Cosmochim. Acta, 214, 172

Zolensky, M., Bland, P., Brown, P., \& Halliday, I. 2006, Meteorites and the Early Solar System II, 869 
E. Dartois et al.: Dome C ultracarbonaceous Antarctic micrometeorites

\section{Appendix A: N/C extended version}

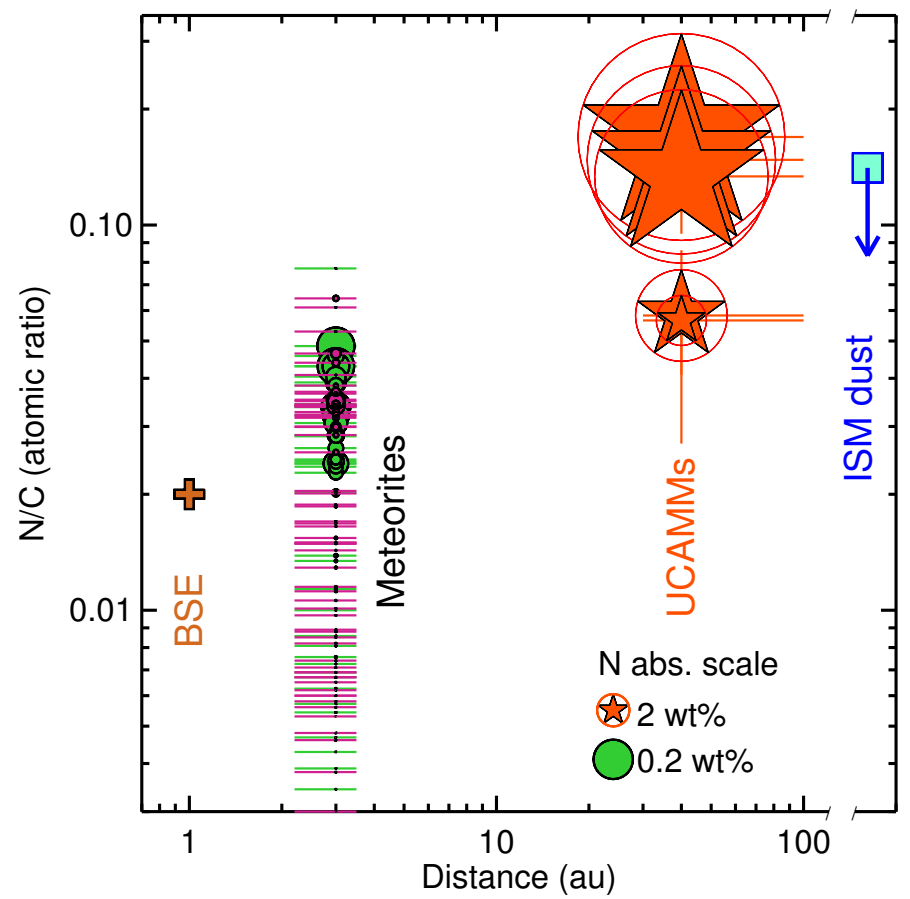

Fig. A.1. UCAMM nitrogen abundances relative to carbon (atomic ratio) are compared to solar system solids: meteorites (Alexander et al. 2007; Kerridge 1985), the bulk silicate Earth (BSE, Bergin et al. 2015), and the interstellar medium (see text for details). The sizes of the symbols for UCAMMs and meteorites give the absolute nitrogen content (in wt \%). UCAMM values are reported in Table B.1.

\section{Appendix B: Measurements summary}

Table B.1. Summary.

\begin{tabular}{|c|c|c|c|c|c|c|c|c|}
\hline UCAMM & DC060919 & DC060594 & DC021119 & DC060443 & DC060718 & DC060741 & DC0609119 & DC060565 \\
\hline \multicolumn{9}{|c|}{ Raman parameters (estimated uncertainty $\sim$ position $\pm 2.5 \mathrm{~cm}^{-1}, F W H M \pm 10 \mathrm{~cm}^{-1}$ ) } \\
\hline \multicolumn{9}{|c|}{$D$ band } \\
\hline Position & 1349.0 & 1358.4 & 1356.6 & 1362.5 & 1362.1 & 1355.9 & 1350.0 & 1342.6 \\
\hline FWHM & 285.4 & 267.0 & 236.2 & 252.6 & 302.8 & 274.8 & 235.3 & 279.6 \\
\hline \multicolumn{9}{|l|}{$G$ band } \\
\hline Position & 1595.9 & 1596.5 & 1589.9 & 1596.8 & 1584.8 & 1585.4 & 1586.2 & 1578.8 \\
\hline FWHM & 90.6 & 89.7 & 150.4 & 100.3 & 125.3 & 147.5 & 143.7 & 120.5 \\
\hline \multicolumn{9}{|c|}{ Infrared measurements } \\
\hline $\int \mathrm{C}=\mathrm{C}\left(\mathrm{cm}^{-1}\right)$ & $17.0 \pm 7.7$ & $27.1 \pm 10.9$ & $43.7 \pm 7.7$ & $13.0 \pm 4.9$ & $16.7 \pm 11.5$ & $22.7 \pm 4.3$ & $12.1 \pm 5.4$ & $33.0 \pm 13.6$ \\
\hline $\int \mathrm{C}=\mathrm{O}\left(\mathrm{cm}^{-1}\right)$ & $2.2 \pm 0.15$ & $7.5 \pm 0.5$ & $29.5 \pm 0.2$ & $1.45 \pm 0.15$ & $2.3 \pm 0.15$ & $8.6 \pm 0.55$ & $5.3 \pm 0.35$ & $9.0 \pm 0.2$ \\
\hline $\mathrm{C}=\mathrm{O}$ position $\left(\mathrm{cm}^{-1}\right)$ & $1685.7 \pm 7.0$ & $1693.6 \pm 7.8$ & $1685.1 \pm 12.5$ & $1687.0 \pm 5.1$ & $1685.1 \pm 3.8$ & $1700.6 \pm 12.7$ & $1705.9 \pm 18.5$ & $1698.8 \pm 7.4$ \\
\hline $\int \mathrm{CH}\left(\mathrm{cm}^{-1}\right)$ & $3.62 \pm 1.21$ & $0.84 \pm 1.09$ & $0.73 \pm 0.12$ & $2.41 \pm 1.54$ & $2.2 \pm 1.9$ & $1.3 \pm 0.6$ & $0.66 \pm 0.43$ & $0.99 \pm 2.15$ \\
\hline $\int$ Silicates $\left(\mathrm{cm}^{-1}\right)$ & $52.77 \pm 15.84$ & $35.93 \pm 10.78$ & $17.75 \pm 5.33$ & $8.33 \pm 2.50$ & $6.85 \pm 2.06$ & $3.95 \pm 1.18$ & $3.95 \pm 1.18$ & $0.02 \pm 0.006$ \\
\hline \multicolumn{9}{|c|}{$\mathrm{N} / \mathrm{C}$ atomic ratios determined with the electron microprobe $\left( \pm 1 \sigma^{a}\right)$} \\
\hline $\mathrm{N}(\mathrm{at} \%)$ & - & $3.3 \pm 1.5$ & $10.2 \pm 2.85$ & - & $12.1 \pm 2.5$ & $5.08 \pm 1.5$ & - & $11.0 \pm 2.8$ \\
\hline $\mathrm{C}(\mathrm{at} \%)$ & - & $58.5 \pm 14.1$ & $76.0 \pm 5.35$ & - & $71.7 \pm 3.9$ & $87.2 \pm 3.3$ & - & $74.7 \pm 6.3$ \\
\hline $\mathrm{N} / \mathrm{C}$ & - & $0.057 \pm 0.029$ & $0.135 \pm 0.039$ & - & $0.170 \pm 0.036$ & $0.058 \pm 0.017$ & - & $0.147 \pm 0.040$ \\
\hline \#measurements & - & 43 & 31 & - & 20 & 13 & - & 22 \\
\hline
\end{tabular}

Notes. ${ }^{(a)}$ The standard deviation includes the measurements errors and intrinsic UCAMM variations of the N \& $\mathrm{C}$ atomic percent in the different zones probed. 